Brian S. Thomson, Mathematics Department, Simon Fraser University,

B. C., Canada V5A 1S6. e-mail: thomson@cs.sfu.ca

\title{
THE SPACE OF DENJOY-PERRON INTEGRABLE FUNCTIONS
}

\begin{abstract}
In the linear space $\mathcal{D} \mathcal{P}[a, b]$ of all Denjoy-Perron integrable functions on an interval $[a, b]$ one wishes to introduce the most natural topology. Herein are some considerations that suggest what topology might be the most natural.
\end{abstract}

\section{Introduction}

Let $\mathcal{D P}[a, b]$ denote the linear space of all Denjoy-Perron integrable functions on an interval $[a, b]$. One frequently studies this space of functions furnished with the norm

$$
\|f\|_{A}=\max _{a \leq x \leq b}|F(x)|
$$

where $F(x)=\int_{a}^{x} f(t) d t$ denotes the indefinite integral of $f$ in the DenjoyPerron sense and, as usual in studies in integration theory, two functions $f$ and $g$ in the space are identified if they have the same indefinite integral (or, equivalently, if they are equal almost everywhere in $[a, b]$ ). The norm in equation (1) is sometimes called the Alexiewicz norm because of the initial study of this space in [1].

Essentially this identifies the space $\mathcal{D P}[a, b]$ with a subspace of the Banach space $C[a, b]$ of continuous functions on $[a, b]$ furnished with the supremum norm, namely the subspace of all the $\mathrm{ACG}_{*}$ functions $F$ in $C[a, b]$ for which $F(a)=0$. Thus, in particular, $\mathcal{D P}[a, b]$ is seen to be an incomplete normed linear space that is first category in itself. In fact, in spite of the category statement, this space is barreled and hence there is a version of the BanachSteinhaus theorem that can be used. See [20] and [22].

The continuous linear functionals on this space can be represented by an integral $f \rightarrow \int_{a}^{b} f(t) g(t) d t$ taken in the Denjoy-Perron sense where $g$ is equivalent to a function of bounded variation ([1], [19]). This should be familiar

Key Words: Denjoy-Perron integral, Alexiewicz norm

Mathematical Reviews subject classification: 26A45, 26A39, 26A46, 26A24.

Received by the editors October 15, 1999 
since the integral can, in this case, be rewritten as a Riemann Stieltjes integral using the integration by parts formula.

One might argue that this norm topology is not the most natural for the space $\mathcal{D P}[a, b]$ since convergence of a sequence of functions $\left\{f_{n}\right\}$ is merely equivalent to uniform convergence of the sequence $\left\{F_{n}\right\}$ of indefinite integrals. This convergence pays little attention to the structure of the Denjoy-Perron integration process.

In contrast consider the usual topology on the space $\mathcal{L}[a, b]$ of Lebesgue integrable functions on an interval $[a, b]$. The norm used there is

$$
\|f\|_{1}=\int_{a}^{b}|f(t)| d t=\operatorname{Var}(F,[a, b])
$$

where, again, $F$ is the indefinite integral of $f$ (now allowed in the Lebesgue sense) and $\operatorname{Var}(F,[a, b])$ is the total variation of $F$ on the interval $[a, b]$. This is a Banach space and the norm (as an integral or as a variation) plays a key role in many investigations of the Lebesgue integral and is an entirely natural object of study.

In this short article we shall study a similar kind of structure in $\mathcal{D P}[a, b]$, and determine its relation to the Alexiewicz norm.

\section{Background Material}

We begin by reminding the reader of the properties of the variation that are needed in a study of the Denjoy-Perron integral. The versions we cite here are not the most general but are tailored to our needs in this article.

Let $F:[a, b] \rightarrow \mathbb{R}$ be a continuous function. Let $[c, d]$ be a closed subinterval and let $E$ be a subset of $[a, b]$. We refer to the expression

$$
\omega_{F}([c, d])=\max _{x \in[c, d]} F(x)-\min _{x \in[c, d]} F(x)
$$

as the oscillation of $F$ on the interval $[c, d]$. The expression

$$
\operatorname{Var}(F, E)=\sup \sum_{i=1}^{p} \omega_{F}\left(\left[a_{i}, b_{i}\right]\right)
$$

is defined by taking the supremum over all nonoverlapping collections of intervals

$$
\left[a_{1}, b_{1}\right],\left[a_{2}, b_{2}\right],\left[a_{3}, b_{3}\right], \ldots,\left[a_{p}, b_{p}\right]
$$

whose endpoints are in the set $E$. This is called the variation of $F$ on $E$. 
A different and closely related notion of variation is as follows. For every positive function $\delta$ on $E$ we define

$$
V(F, E ; \delta)=\sup \sum_{i=1}^{p}\left|F\left(a_{i}\right)-F\left(b_{i}\right)\right|
$$

where the supremum is taken over all nonoverlapping collections of subintervals of $[a, b]$

$$
\left\{\left[a_{1}, b_{1}\right],\left[a_{2}, b_{2}\right],\left[a_{3}, b_{3}\right], \ldots,\left[a_{p}, b_{p}\right]\right\}
$$

for which there is a collection of points $\xi_{i} \in E \cap\left[a_{i}, b_{i}\right](i=1,2,3, \ldots p)$ with $b_{i}-a_{i}<\delta\left(\xi_{i}\right)$. Then we define $V(F, E)=\inf V(F, E ; \delta)$ where the infimum is taken over all positive functions $\delta$ on $E$. These three expressions $\operatorname{Var}(F, E)$, $V(F, E ; \delta)$ and $V(F, E)$ define the variational concepts that can be used to express the nature of the Denjoy-Perron integration process.

Most of the following facts are well known and can be found in a variety of sources (e.g., [2], [3], [9], [13], [16], [17], [23], [24]). We have indicated the proofs for any statements that may be less well known or are not often used.

2.1. $F \rightarrow \operatorname{Var}(F, E)$ is a seminorm, i.e.,

$$
\operatorname{Var}\left(F_{1}+F_{2}, E\right) \leq \operatorname{Var}\left(F_{1}, E\right)+\operatorname{Var}\left(F_{2}, E\right)
$$

and, for any $c \in \mathbb{R}$,

$$
\operatorname{Var}(c F, E)=|c| V(F, E) .
$$

2.2. For any subset $E$ of $[a, b]$ and any continuous function $F$

$$
\operatorname{Var}(F, E)=\operatorname{Var}(F, \bar{E}) .
$$

2.3. For any two subsets $E_{1}$ and $E_{2}$ of $[a, b]$

$$
\operatorname{Var}\left(F, E_{1} \cup E_{2}\right) \leq 2\left\{\operatorname{Var}\left(F, E_{1}\right)+\operatorname{Var}\left(F, E_{2}\right)+\omega_{F}([a, b])\right\} .
$$

Proof. Let there be given a nonoverlapping collection of intervals

$$
\left[a_{1}, b_{1}\right],\left[a_{2}, b_{2}\right],\left[a_{3}, b_{3}\right], \ldots,\left[a_{p}, b_{p}\right]
$$

whose endpoints are in the set $E_{1} \cup E_{2}$. We can split the collection into four subcollections: in the first, place those intervals $\left[a_{i}, b_{i}\right]$ for which $a_{i}, b_{i} \in E_{1}$; in the second, place those remaining for which $a_{i}, b_{i} \in E_{2}$; in the third, place those remaining for which $a_{i} \in E_{1}, b_{i} \in E_{2}$; and in the final collection place those remaining and it will be the case that each $a_{i} \in E_{2}, b_{i} \in E_{1}$. 
We now compute an upper bound for the sums $\sum_{i=1}^{p} \omega_{F}\left(\left[a_{i}, b_{i}\right]\right)$ by splitting across the four collections. If these are taken just over the first and second collections, then they are clearly bounded by $\operatorname{Var}\left(F, E_{1}\right)$ and $\operatorname{Var}\left(F, E_{2}\right)$ respectively. Consider now the sums taken over the third collection. To be specific let $\left[\alpha_{i}, \beta_{i}\right]$ for $i=1,2, \ldots q$ denote this collection. We may assume that $\alpha_{1}<\beta_{1} \leq \alpha_{2}<\beta_{2} \leq \ldots \alpha_{q}<\beta_{q}$ by relabeling if necessary. Here $\alpha_{i} \in E_{1}$ and $\beta_{i} \in E_{2}$. Note that

$$
\sum_{i=1}^{q} \omega_{F}\left(\left[\alpha_{i}, \beta_{i}\right]\right) \leq \sum_{i=1}^{q-1} \omega_{F}\left(\left[\alpha_{i}, \alpha_{i+1}\right]\right)+\omega_{F}\left(\left[\alpha_{q}, \beta_{q}\right]\right) .
$$

In particular an upper bound for these sums is then given by

$$
\operatorname{Var}\left(F, E_{1}\right)+\omega_{F}([a, b]) .
$$

Similarly an upper bound for the sums of the fourth type would be given by

$$
\operatorname{Var}\left(F, E_{2}\right)+\omega_{F}([a, b]) .
$$

Putting these four upper bounds together then yields the upper bound (3) of the lemma.

2.4. For any function $F$, the total variation of $F$ on $[a, b]$ is equal to

$$
\operatorname{Var}(F,[a, b])=V(F,[a, b])=V(F,[a, b], \delta)
$$

for any $\delta$. (In particular these are finite precisely when $F$ has bounded variation on $[a, b])$.

2.5. $E \rightarrow V(F, E)$ is a metric outer measure, i.e., the function

$$
F^{*}(E)=V(F, E)
$$

defined on all subsets of $[a, b]$ is an outer measure for which all Borel sets are measurable.

2.6. If $F$ is a continuous function on $[a, b]$ and $C$ is countable then

$$
V(F, C)=0
$$

2.7. If $F$ is a continuous function on $[a, b]$ and $E \subset[a, b]$ is a closed set then

$$
V(F, E) \leq 2 \operatorname{Var}(F, E) .
$$


Proof. We assume $E$ is nonempty. Let $\alpha=\inf E$ and $\beta=\sup E$. Let $C$ denote the countable collection of all points in $E$ that are isolated on one side at least and let $D=E \backslash C$. Choose any $\delta$ so that if $x \in D$ then $\delta(x)<x-\alpha$ and $\delta(x)<\beta-x$.

We now estimate $V(F, D, \delta)$. Let $\left[a_{1}, b_{1}\right],\left[a_{2}, b_{2}\right],\left[a_{3}, b_{3}\right], \ldots\left[a_{n}, b_{n}\right]$ be any nonoverlapping collection of subintervals of $[a, b]$ for which there is a collection of points $\xi_{i} \in D(i=1,2,3, \ldots n)$ with $b_{i}-a_{i}<\delta\left(\xi_{i}\right)$. (Assume that the intervals are naturally ordered from left to right.) We use the points

$$
\alpha=\xi_{0}<\xi_{1}<\xi_{2}<\cdots<\xi_{n+1}=\beta
$$

from $E$ and note that each interval $\left[\xi_{i}, \xi_{i+1}\right]$ meets at most two of the intervals $\left\{\left[a_{i}, b_{i}\right]\right\}$. These intervals $\left[\xi_{i}, \xi_{i+1}\right]$ must cover the intervals $\left\{\left[a_{i}, b_{i}\right]\right\}$. Consequently

$$
\sum_{i=1}^{n}\left|F\left(b_{i}\right)-F\left(a_{i}\right)\right| \leq 2 \sum_{i=1}^{p} \omega_{F}\left(\left[\xi_{i}, \xi_{i+1}\right]\right) \leq 2 \operatorname{Var}(F, E) .
$$

It follows that

$$
V(F, D) \leq V(F, D, \delta) \leq 2 \operatorname{Var}(F, E) .
$$

But from 2.5 and $\mathbf{2 . 6}$ we see that $V(F, C)=0$ and

$$
V(F, E)=V(F, D)+V(F, C) .
$$

Thus assertion (4) follows now from assertion (5) and (6).

2.8. A continuous function $F$ on $[a, b]$ is $V B G_{*}$ if and only if there exists an increasing sequence of closed sets $\left\{E_{n}\right\}$ covering $[a, b]$ such that each $\operatorname{Var}\left(F, E_{n}\right)$ is finite.

Proof. The usual definition of $\mathrm{VBG}_{*}$ does not assume this form, but for continuous functions this version can be easily checked to be equivalent to the one in Saks [17]. For if $F$ is $\mathrm{VBG}_{*}$ on $[a, b]$ there is a sequence of sets $\left\{C_{n}\right\}$ covering $[a, b]$ and each $\operatorname{Var}\left(F, C_{n}\right)$ is finite. Since $F$ is continuous here we may take

$$
E_{n}=\overline{C_{1}} \cup \overline{C_{2}} \cup \cdots \cup \overline{C_{n}}
$$

and use assertions $\mathbf{2 . 2}$ and $\mathbf{2 . 3}$ to conclude that each $\operatorname{Var}\left(F, E_{n}\right)$ is finite.

2.9. $F \rightarrow V(F, E)$ is a seminorm, i.e.,

$$
V\left(F_{1}+F_{2}, E\right) \leq V\left(F_{1}, E\right)+V\left(F_{2}, E\right)
$$

and, for all $c \in \mathbb{R}, V(c F, E)=|c| V(F x, E)$. 
2.10. A continuous function $F$ is $V B G_{*}$ on $[a, b]$ if and only if the outer measure $F^{*}$ is $\sigma$-finite on $[a, b]$.

2.11. A continuous function $F$ is $A C G_{*}$ if and only if $F^{*}$ vanishes on all closed subsets of $[a, b]$ of Lebesgue measure zero.

2.12. The relation $F(x)=\int_{a}^{b} f(t) d t$ holds in the sense of the Denjoy-Perron integral if and only if $F(a)=0, F^{\prime}(x)=f(x)$ almost everywhere and $F$ is $A C G_{*}$ on $[a, b]$.

2.13. If $F^{\prime}$ exists on a Borel set $E$ then $V(F, E)=\int_{E}\left|F^{\prime}(t)\right| d t$ where the integral is in the Lebesgue sense.

\section{The Space $\mathcal{D P}\left(\left\{E_{n}\right\}\right)$}

Let $f$ be a function that is Denjoy-Perron integrable on an interval $[a, b]$. Then, writing $F(x)=\int_{a}^{x} f(t) d t$ we know that there exists an increasing sequence of closed sets $\left\{E_{n}\right\}$ covering $[a, b]$ such that

$$
\operatorname{Var}\left(F, E_{n}\right)<\infty
$$

for each $n=1,2,3, \ldots$. Because of this we are led to define, for each fixed family $\left\{E_{n}\right\}$ forming an increasing sequence of closed sets covering $[a, b]$, the family $\mathcal{D} \mathcal{P}\left(\left\{E_{n}\right\}\right)$ of all functions $f$ in $\mathcal{D P}[a, b]$ for which (7) holds. This is clearly a linear subspace of $\mathcal{D} \mathcal{P}[a, b]$. The functions $p_{n}$ defined as

$$
p_{n}(f)=\operatorname{Var}\left(F, E_{n}\right)
$$

form an increasing sequence of seminorms on $\mathcal{D P}\left(\left\{E_{n}\right\}\right)$ that serve to define a locally convex topology. This topology is the same as that provided by the metric

$$
d(f, g)=\sum_{n=1}^{\infty} \frac{1}{2^{n}} \frac{p_{n}(f-g)}{1+p_{n}(f-g)} .
$$

It is this topology we shall impose on $\mathcal{D P}\left(\left\{E_{n}\right\}\right)$. (Recall that, throughout, we identify two functions in these spaces if they have the same indefinite integral.)

Note that if $f$ is a member of the space $\mathcal{D P}\left(\left\{E_{n}\right\}\right)$ then, because of $\mathbf{2 . 7}$ and 2.13,

$$
\int_{E_{n}}|f(t)| d t=V\left(F, E_{n}\right) \leq 2 \operatorname{Var}\left(F, E_{n}\right)<\infty
$$

so that $f$ is also Lebesgue integrable on each set in the sequence $\left\{E_{n}\right\}$. (The converse is not true: a function $f$ could well be Lebesgue integrable on each set in the sequence $\left\{E_{n}\right\}$ and yet fail to be Denjoy-Perron integrable on $[a, b]$.) 
Theorem 3.1. Let $\left\{E_{n}\right\}$ be an increasing sequence of closed sets covering $[a, b]$. Then $\mathcal{D P}\left(\left\{E_{n}\right\}\right)$ is a metrizable, complete, locally convex topological vector space.

Proof. A countable family of seminorms always imposes a topology that is locally convex and metrizable, thus it is only the completeness assertion that needs to be proved.

Let $\left\{f_{k}\right\}$ be a sequence of functions in $\mathcal{D P}\left(\left\{E_{n}\right\}\right)$ that is assumed to be Cauchy relative to each of the seminorms in (8). If $F$ denotes the indefinite integral of any element $f$ of the space we observe that, for any $n$ sufficiently large so that $a, b \in E_{n}, \omega_{F}([a, b]) \leq \operatorname{Var}\left(F, E_{n}\right)=p_{n}(f)$. Consequently, if $\left\{F_{k}\right\}$ denotes the corresponding sequence of indefinite integrals for the given sequence $\left\{f_{k}\right\}$ we see that $\left\{F_{k}\right\}$ is uniformly Cauchy on $[a, b]$ and so convergent to a continuous function $F$ on $[a, b]$. Let $\epsilon>0$ and, fixing $n$, choose an integer $K$ so large that $\operatorname{Var}\left(F_{k}-F_{j}, E_{n}\right)=p_{n}\left(f_{k}-f_{j}\right)<\epsilon$ if $j, k \geq K$.

Fix $n$ and consider the sum

$$
\sum_{i=1}^{p} \omega_{F_{k}-F_{j}}\left(\left[a_{i}, b_{i}\right]\right) \leq \operatorname{Var}\left(F_{k}-F_{j}, E_{n}\right)<\epsilon
$$

where

$$
\left[a_{1}, b_{1}\right],\left[a_{2}, b_{2}\right],\left[a_{3}, b_{3}\right], \ldots,\left[a_{p}, b_{p}\right]
$$

is any nonoverlapping collection of subintervals of $[a, b]$ whose endpoints are in the set $E_{n}$. By holding $k \geq K$ fixed and letting $j \rightarrow \infty$ in the inequality (9) we obtain that $\sum_{i=1}^{p} \omega_{F_{k}-F}\left(\left[a_{i}, b_{i}\right]\right) \leq \epsilon$. It follows that $\operatorname{Var}\left(F_{k}-F, E_{n}\right) \leq \epsilon$ for all $k \geq K$. In particular we conclude for each $n$ that

$$
\lim _{k \rightarrow \infty} \operatorname{Var}\left(F_{k}-F, E_{n}\right)=0 .
$$

Note, too, that each $\operatorname{Var}\left(F, E_{n}\right)<\infty$.

We now show that $F$ is $\mathrm{ACG}_{*}$. Fix $n, \epsilon>0$ as above, and fix a closed set $Z \subset[a, b]$ of Lebesgue measure zero. We know from 2.11 that $V\left(F_{K}, Z\right)=0$. But from 2.7 we have

$V\left(F, Z \cap E_{n}\right) \leq V\left(F_{K}, Z \cap E_{n}\right)+V\left(F_{K}-F, Z \cap E_{n}\right) \leq 2 \operatorname{Var}\left(F_{k}-F, E_{n}\right) \leq \epsilon$.

Since $\epsilon>0$ is an arbitrary positive number it follows that $V\left(F, Z \cap E_{n}\right)=0$. Now using the measure property $\mathbf{2 . 5}$ of the variation we have

$$
V(F, Z) \leq \sum_{n=1}^{\infty} V\left(F, E_{n} \cap Z\right)=0
$$


Since $Z$ can be any closed set of measure zero we conclude, again using $\mathbf{2 . 1 1}$, that $F$ is $\mathrm{ACG}_{*}$.

Since $F$ is $\mathrm{ACG}_{*}$ it is differentiable almost everywhere and is the indefinite integral in the Denjoy-Perron sense of its derivative. Let $f=F^{\prime}$ and we see that $f$ belongs to $\mathcal{D P}[a, b]$. Indeed since we have seen that $\operatorname{Var}\left(F, E_{n}\right)<\infty$ for each $n$ we know that $f$ belongs to $\mathcal{D P}\left(\left\{E_{n}\right\}\right)$. ¿From the assertion (10) we conclude that the Cauchy sequence $\left\{f_{k}\right\}$ converges in the space $\mathcal{D P}\left(\left\{E_{n}\right\}\right)$ to the function $f$. This establishes that this space is complete and the proof is done.

Example 1. $\mathcal{L}[a, b]$ is precisely the space $\mathcal{D P}\left(\left\{E_{n}\right\}\right)$ for an appropriate choice of $\left\{E_{n}\right\}$ (namely each $E_{n}=[a, b]$ ).

Example 2. Consider the sequence of sets $E_{n}=\{a\} \cup[a+1 / n, b]$. The space $\mathcal{D P}\left(\left\{E_{n}\right\}\right)$ forms a subspace of the space of Denjoy-Perron integrable functions on the interval $[a, b]$. This can be described in another way. A function $f$ belongs to this space if and only if $f$ is Denjoy-Perron integrable on $[a, b]$ and Lebesgue integrable on every interval $[a+\epsilon, b]$ for $\epsilon>0$. Or, equivalently, if and only if $f$ is Lebesgue integrable on every interval $[a+\epsilon, b]$ for $\epsilon>0$ and $\lim _{t \rightarrow a+} \int_{t}^{b} f(x) d x$ exists.

We now discuss the continuous linear functionals on the spaces $\mathcal{D P}\left(\left\{E_{n}\right\}\right)$. Note that the theorem here does not offer a characterization. For different choices of $\left\{E_{n}\right\}$ there will be different restrictions on the function $g$ in the theorem. For Example 1 the function $g$ can be any bounded measurable function on $[a, b]$ while for Example 2 there would be restrictions on $g$.

Theorem 3.2. Let $\left\{E_{n}\right\}$ be an increasing sequence of closed sets covering $[a, b]$. Then if $\Gamma$ is a continuous linear functional on the space $\mathcal{D P}\left(\left\{E_{n}\right\}\right)$ there exists a bounded, measurable function $g$ on $[a, b]$ so that

$$
\Gamma(f)=\int_{a}^{b} f(t) g(t) d t \quad\left(f \in \mathcal{D} \mathcal{P}\left(\left\{E_{n}\right\}\right)\right)
$$

where the integral exists in the Denjoy-Perron sense.

Proof. It suffices to show the existence of a measurable function $g$ on $[a, b]$ with this property since, in order for this integral to exist for all Lebesgue integrable functions $f$ it is clear that $g$ must be bounded. Let $\mathcal{S}$ denote the collection of all intervals $(c, d) \subset(a, b)$ so that there exists a measurable function $g$ on $[c, d]$ with the property that

$$
\Gamma\left(f^{*}\right)=\int_{c}^{d} f(t) g(t) d t \quad\left(f \in \mathcal{D} \mathcal{P}\left(\left\{E_{n}\right\}\right)\right)
$$


where the integral exists in the Denjoy-Perron sense and where $f^{*}$ is the function defined on $[a, b]$ so as to agree with $f$ on $[c, d]$ and to vanish elsewhere. Note that if $f \in \mathcal{D P}\left(\left\{E_{n}\right\}\right)$ then necessarily $f^{*} \in \mathcal{D} \mathcal{P}\left(\left\{E_{n}\right\}\right)$ so that $\Gamma\left(f^{*}\right)$ is always defined.

We claim that the interval $(a, b)$ belongs to $\mathcal{S}$ and, hence, that the theorem is proved. In order to obtain a contradiction let us suppose that this is not the case.

If it is not true that $(a, b) \in \mathcal{S}$ then let $E=[a, b] \backslash \bigcup_{(c, d) \in \mathcal{S}}(c, d)$. If $(a, b) \in \mathcal{S}$ is false then $E$ is a nonempty closed subset of $[a, b]$. By the Baire category theorem, there is an integer $M$ and an interval $(c, d)$ so that

$$
E \cap(c, d)=E_{M} \cap(c, d) \neq \emptyset .
$$

We will show that it then follows that $(c, d) \in \mathcal{S}$ which is the desired contradiction since $E$ cannot contain any point of an interval belonging to $\mathcal{S}$.

Step 1 . We first observe that $\mathcal{S}$ has an hereditary property: if any interval $\left(c^{\prime}, d^{\prime}\right)$ belongs to $\mathcal{S}$ then so too does every subinterval $\left(c^{\prime \prime}, d^{\prime \prime}\right) \subset\left(c^{\prime}, d^{\prime}\right)$. This is easily checked.

Step 2. Now let us show that every subinterval $\left(c^{\prime}, d^{\prime}\right)$ that is a component of $(a, b) \backslash E$ belongs to $\mathcal{S}$.

Step 2(a). Consider first any interval $\left[c^{\prime \prime}, d^{\prime \prime}\right] \subset\left(c^{\prime}, d^{\prime}\right)$. By a compactness argument $\left[c^{\prime \prime}, d^{\prime \prime}\right]$ is covered by finitely many intervals from $\mathcal{S}$. We argue that this will require that $\left(c^{\prime \prime}, d^{\prime \prime}\right) \in \mathcal{S}$.

To illustrate the argument suppose that $\left(c^{\prime \prime}, d^{\prime \prime}\right)$ is covered by two intervals belonging to $\mathcal{S}$, say $\left(x, y^{\prime}\right),\left(y^{\prime \prime}, z\right)$ and $x \leq c^{\prime \prime}<y^{\prime \prime}<y^{\prime}<d^{\prime \prime} \leq z$. Then, for any choice of $y^{\prime \prime}<y<y^{\prime}$, by the hereditary property of $\mathcal{S}$ both intervals $\left(c^{\prime \prime}, y\right)$ and $\left(y, d^{\prime \prime}\right)$ belong to $\mathcal{S}$. Thus there are functions $g_{1}$ and $g_{2}$ corresponding to the two adjacent intervals $\left(c^{\prime \prime}, y\right)$ and $\left(y, d^{\prime \prime}\right)$ (respectively) and which verify that $\left(c^{\prime \prime}, y\right) \in \mathcal{S}$ and $\left(y, d^{\prime \prime}\right) \in \mathcal{S}$. Then the function $g$ defined on $\left(c^{\prime \prime}, d^{\prime \prime}\right)$ by setting $g(t)=g_{1}(t)$ for $t \in\left(c^{\prime \prime}, y\right)$ and $g(t)=g_{2}(t)$ for $t \in\left(y, d^{\prime \prime}\right)$ supplies a verification that $\left(c^{\prime \prime}, d^{\prime \prime}\right) \in \mathcal{S}$. This requires only the additivity of the functional $\Gamma$. A similar argument will handle any other situations in which a finite collection of intervals from $\mathcal{S}$ covers $\left(c^{\prime \prime}, d^{\prime \prime}\right)$.

Step 2(b). We proceed now to show that every subinterval $\left(c^{\prime}, d^{\prime}\right)$ complementary to $E$ in $(a, b)$ belongs to $\mathcal{S}$. Fix $\left(c^{\prime}, d^{\prime}\right)$ as a component interval of the set $(a, b) \backslash E$. Take any sequence of positive numbers $\epsilon_{n} \rightarrow 0$ and consider the subintervals $\left(c^{\prime}+\epsilon_{n}, d^{\prime}-\epsilon_{n}\right)$. Since each belongs to $\mathcal{S}$ (by the step 2(a) just proved) there is a measurable function $g_{n}$ defined on each $\left[c^{\prime}+\epsilon_{n}, d^{\prime}-\epsilon_{n}\right]$ so that

$$
\Gamma\left(f_{n}\right)=\int_{c+\epsilon_{n}}^{d-\epsilon_{n}} f(t) g_{n}(t) d t \quad\left(f \in \mathcal{D P} \mathcal{P}\left(\left\{E_{n}\right\}\right)\right)
$$


where the integral exists in the Denjoy-Perron sense and where $f_{n}$ is the function defined on $[a, b]$ so as to agree with $f$ on $\left(c^{\prime}+\epsilon_{n}, d^{\prime}-\epsilon_{n}\right)$ and to vanish elsewhere. Since, up to equivalence, there is only one such function and these intervals expand to cover all of $\left(c^{\prime}, d^{\prime}\right)$, we may take it that there is a single measurable function $g$ defined on $\left[c^{\prime}, d^{\prime}\right]$ so that this equation (11) holds for all $n$ with $g$ replacing $g_{n}$.

We notice now that if $f^{*}$ is the function defined on $[a, b]$ so as to agree with $f$ on $\left[c^{\prime}, d^{\prime}\right]$ and to vanish elsewhere, then $p_{m}\left(f_{n}-f^{*}\right) \rightarrow 0$ for every $m$ as $n \rightarrow \infty$. Let us check this. To compute $p_{m}\left(f_{n}-f^{*}\right)$ we note that the function $f^{*}-f_{n}$ is identical to $f$ on $\left[c^{\prime}, c^{\prime}+\epsilon_{n}\right]$ and $\left[d^{\prime}-\epsilon_{n}, d^{\prime}\right]$ and vanishes elsewhere. In particular then, for any $m$,

$$
p_{m}\left(f_{n}-f^{*}\right) \leq \operatorname{Var}\left(F, E_{m} \cap\left[c^{\prime}, c^{\prime}+\epsilon_{n}\right]\right)+\operatorname{Var}\left(F, E_{m} \cap\left[d^{\prime}-\epsilon_{n}, d^{\prime}\right]\right) .
$$

Since $f$ is a member of the space $\mathcal{D P}\left(\left\{E_{n}\right\}\right)$ it follows that $\operatorname{Var}\left(F, E_{m}\right)<\infty$. Since $F$ is also continuous it must be true that

$$
\operatorname{Var}\left(F, E_{m} \cap\left[c^{\prime}, c^{\prime}+\epsilon_{n}\right]\right) \rightarrow 0
$$

and

$$
\operatorname{Var}\left(F, E_{m} \cap\left[d^{\prime}-\epsilon_{n}, d^{\prime}\right]\right) \rightarrow 0
$$

as $n \rightarrow \infty$. It follows from these two assertions and (12) that $p_{m}\left(f_{n}-f^{*}\right) \rightarrow 0$ as $n \rightarrow \infty$.

Thus $f_{n} \rightarrow f^{*}$ in the space $\mathcal{D P}\left(\left\{E_{n}\right\}\right)$ and so, since $\Gamma$ is continuous,

$$
\Gamma\left(f_{n}\right) \rightarrow \Gamma(f) .
$$

This means, because of (11), that the limit $\lim _{n \rightarrow \infty} \int_{c^{\prime}+\epsilon_{n}}^{d^{\prime}-\epsilon_{n}} f(t) g(t) d t$ exists. As this is true for all such sequences $\left\{\epsilon_{n}\right\}$ it follows, from the Cauchy property of the Denjoy-Perron integral, that $f g$ is Denjoy-Perron integrable on $\left(c^{\prime}, d^{\prime}\right)$. It also follows that

$$
\Gamma\left(f^{*}\right)=\lim _{n \rightarrow \infty} \Gamma\left(f_{n}\right)=\int_{c^{\prime}}^{d^{\prime}} f(t) g(t) d t .
$$

But this identity verifies that $\left(c^{\prime}, d^{\prime}\right) \in \mathcal{S}$ as we wished to prove.

Step 3. We now check that there exists a measurable function $k$ on $E \cap[c, d]$ so that

$$
\Gamma\left(f_{E}\right)=\int_{E} f(t) k(t) d t \quad\left(f \in \mathcal{D} \mathcal{P}\left(\left\{E_{n}\right\}\right)\right)
$$

where the integral exists in the Lebesgue sense and where $f_{E}$ denotes the function defined on $[a, b]$ so as to agree with $f$ on $E$ and to vanish on $[a, b] \backslash E$. 
To obtain the function $k$ in (13) we construct a continuous linear functional on the space $\mathcal{L}(E \cap[c, d])$ of Lebesgue integrable functions on the set $E \cap[c, d]$. Let $h$ be any function that is Lebesgue integrable on $E \cap[c, d]$ and use $h^{*}$ to denote the function defined on $[a, b]$ that agrees with $h$ on $E \cap[c, d]$ and vanishes elsewhere. Clearly $h^{*}$ must be Lebesgue integrable on $[a, b]$. Moreover if $H^{*}$ is its indefinite integral then $H^{*}$ is constant on each interval complementary to $E \cap[c, d]$ in $[a, b]$. Thus we can compute, using $\mathbf{2 . 4}$ and $\mathbf{2 . 1 3}$, that

$$
\operatorname{Var}\left(H^{*}, E \cap[c, d]\right)=\operatorname{Var}\left(H^{*},[a, b]\right)=\int_{a}^{b}\left|h^{*}(t)\right| d t=\int_{E \cap[c, d]}|h(t)| d t .
$$

We also note that

$$
p_{m}\left(h^{*}\right)=\int_{E \cap[c, d]}|h(t)| d t
$$

for all $m \geq M$, since $E_{m}$ contains $E \cap[c, d]$.

Now we consider the functional $\Gamma^{\prime}$ defined on the space $\mathcal{L}(E \cap[c, d])$ equipped with the usual norm (see equation $(2)$ ) where $\Gamma^{\prime}$ is defined by the identity by

$$
\Gamma^{\prime}(h)=\Gamma\left(h^{*}\right) \quad(h \in \mathcal{L}(E \cap[c, d])) .
$$

Clearly $\Gamma^{\prime}(h)$ is defined for every $h \in \mathcal{L}(E \cap[c, d])$ and is linear. We now check that $\Gamma^{\prime}$ is continuous on that space. If $h_{n} \rightarrow h$ in the sense of the norm then

$$
\int_{E \cap[c, d]}\left|h_{n}(t)-h(t)\right| d t \rightarrow 0
$$

But from (14) this means that $h_{n}^{*} \rightarrow h^{*}$ in the space $\mathcal{D} \mathcal{P}\left(\left\{E_{n}\right\}\right)$. Since $\Gamma$ is continuous on the space $\mathcal{D} \mathcal{P}\left(\left\{E_{n}\right\}\right)$ this then requires that $\Gamma\left(h_{n}^{*}\right) \rightarrow \Gamma\left(h^{*}\right)$. From (15) it follows that $\Gamma^{\prime}\left(h_{n}\right) \rightarrow \Gamma^{\prime}(h)$ as we needed to show to prove that $\Gamma^{\prime}$ is continuous.

But any continuous linear functional on $\mathcal{L}(E \cap[c, d])$ has a representation as an integral $\Gamma^{\prime}(h)=\int_{E \cap[c, d]} h(t) k(t) d t$ where the integral is in the Lebesgue sense and where $k$ is some measurable function on $E \cap[c, d]$. (See, for example, $[7$, p. 588].)

If $f \in \mathcal{D P}\left(\left\{E_{n}\right\}\right)$ then $f$ is Lebesgue integrable on each $E_{n}$ and so, in particular, also Lebesgue integrable on $E \cap[c, d]$. Thus using $h=f_{E}$ we have

$$
\Gamma\left(f_{E}\right)=\int_{E \cap(c, d)} f(t) k(t) d t \quad\left(f \in \mathcal{D} \mathcal{P}\left(\left\{E_{n}\right\}\right)\right)
$$

exactly as needed for the representation in (13). 
Step 4. Now let $\left\{\left(a_{i}, b_{i}\right)\right\}$ denote the sequence of intervals complementary to $E$ in $(c, d)$. Since each interval $\left(a_{i}, b_{i}\right)$ belongs to $\mathcal{S}$ by Step 2, there is a function $g_{i}$ which can be used to verify that $\left(a_{i}, b_{i}\right) \in \mathcal{S}$. We will show that, for every $f \in \mathcal{D P}\left(\left\{E_{n}\right\}\right)$,

$$
\Gamma\left(f^{*}\right)=\int_{E} f(t) k(t) d t+\sum_{i=1}^{\infty} \int_{a_{i}}^{b_{i}} f(t) g_{i}(t) d t
$$

where $f^{*}$ is the function defined on $[a, b]$ so as to agree with $f$ on $[c, d]$ and to vanish elsewhere.

Define $f_{n}$ to be equal to $f$ on $E$ and on each of the intervals $\left(a_{i}, b_{i}\right)$ for $i \leq n$ and to vanish elsewhere. Note, from the additivity of $\Gamma$, that

$$
\Gamma\left(f_{n}\right)=\int_{E} f(t) k(t) d t+\sum_{i=1}^{n} \int_{a_{i}}^{b_{i}} f(t) g_{i}(t) d t
$$

We shall show that $p_{m}\left(f_{n}-f^{*}\right) \rightarrow 0$ for every $m \geq M$ as $n \rightarrow \infty$. Fix $m$ and consider $p_{m}\left(f_{n}-f^{*}\right)$ The function $f_{n}-f^{*}$ vanishes on $[c, d]$ everywhere except on the intervals $\left(a_{i}, b_{i}\right)$ for $i>n$ where it has the same values as $-f$. Consequently

$$
p_{m}\left(f_{n}-f^{*}\right) \leq \sum_{i>n} \operatorname{Var}\left(F, E_{m} \cap\left(a_{i}, b_{i}\right)\right) .
$$

But $\operatorname{Var}\left(F, E_{m}\right)<\infty$ and this requires

$$
\sum_{i=1}^{\infty} \operatorname{Var}\left(F, E_{m} \cap\left(a_{i}, b_{i}\right)\right)<\infty
$$

and consequently

$$
\lim _{n \rightarrow \infty} p_{m}\left(f_{n}-f^{*}\right) \leq \lim _{n \rightarrow \infty} \sum_{i>n} \operatorname{Var}\left(F, E_{m} \cap\left(a_{i}, b_{i}\right)\right)=0 .
$$

Thus $f_{n} \rightarrow f^{*}$ in the space $\mathcal{D P}\left(\left\{E_{n}\right\}\right)$ and so, since $\Gamma$ is continuous,

$$
\Gamma\left(f_{n}\right) \rightarrow \Gamma(f) .
$$

From (17) and the identity (16) we obtain

$$
\lim _{n \rightarrow \infty} \Gamma\left(f_{n}\right)=\int_{E} f(t) h(t) d t+\sum_{i=1}^{\infty} \int_{a_{i}}^{b_{i}} f(t) g_{i}(t) d t
$$

where the sum must exist. This completes step 4 . 
In particular note that, since the order of the components is immaterial the series converges in any rearrangement and so

$$
\sum_{i=1}^{\infty}\left|\int_{a_{i}}^{b_{i}} f(t) g_{i}(t) d t\right|<\infty .
$$

We actually need more. We need that

$$
\sum_{i=1}^{\infty}\left|\int_{\alpha_{i}}^{\beta_{i}} f(t) g_{i}(t) d t\right|<\infty
$$

for any choices of $\left(\alpha_{i}, \beta_{i}\right) \subset\left(a_{i}, b_{i}\right)$. But, in fact, this must be true since the same argument can apply by replacing the function $f$ under consideration by a new function $f_{1}$ that agrees with $f$ everywhere except on the intervals $\left(a_{i}, \alpha_{i}\right)$ and $\left(\beta_{i}, b_{i}\right)$ where it vanishes. The new function $f_{1}$ is also in the space and, if the same arguments are repeated applied to $f_{1}$ it will be obtained that the series (19) converges absolutely for any choices of $\left(\alpha_{i}, \beta_{i}\right) \subset\left(a_{i}, b_{i}\right)$.

Step 6. Define $g$ on $(c, d)$ so that $g(t)=k(t)$ for $t \in E$ and $g(t)=g_{i}(t)$ for $t \in\left(a_{i}, b_{i}\right)$. Clearly $g$ is measurable. Recall that $k$ has been defined in Step 3. It now follows, from (19) and the Harnack property of the Denjoy-Perron integral, that $f g$ is Denjoy-Perron integrable on $(c, d)$. From (17) and (18) we obtain that

$$
\Gamma(f)=\int_{E} f(t) h(t) d t+\sum_{i=1}^{\infty} \int_{a_{i}}^{b_{i}} f(t) g_{i}(t) d t=\int_{c}^{d} f(t) g(t) d t .
$$

This identity verifies that $(c, d) \in \mathcal{S}$. Since this is the desired contraction the proof is complete.

\section{The Space $\mathcal{D P}[a, b]$}

The most natural topology on the space $\mathcal{D P}[a, b]$, it may perhaps now be argued, is to take the finest locally convex topology such that each of the canonical injections from the spaces $\mathcal{D P}\left(\left\{E_{n}\right\}\right)$ into $\mathcal{D} \mathcal{P}[a, b]$ is continuous. Such a topology is sometimes called an inductive limit topology although in most applications the directed set of subspaces is countable.

We show that this topology is in fact equivalent to that given by the Alexiewicz norm.

Theorem 4.1. The finest convex topology on $\mathcal{D} \mathcal{P}[a, b]$ such that each of the canonical injections from the spaces $\mathcal{D P}\left(\left\{E_{n}\right\}\right)$ into $\mathcal{D P}[a, b]$ is continuous is the norm topology given by the Alexiewicz norm. 
Proof. Let $\tau$ denote the inductive limit topology, i.e., the finest convex topology on $\mathcal{D} \mathcal{P}[a, b]$ such that each of the canonical injections from the spaces $\mathcal{D P}\left(\left\{E_{n}\right\}\right)$ into $\mathcal{D} \mathcal{P}[a, b]$ is continuous. Let $\tau_{A}$ denote the topology generated by the Alexiewicz norm.

Let us note one simple fact. The topology $\tau$ is finer than the topology $\tau_{A}$. For if $G$ is the identity mapping from $(\mathcal{D P}[a, b], \tau) \rightarrow\left(\mathcal{D P}[a, b], \tau_{A}\right)$ then we know, from properties of inductive limits, that $G$ is continuous if and only if the restricted mapping from each space $\mathcal{D P}\left(\left\{E_{n}\right\}\right)$ into $\left(\mathcal{D P}[a, b], \tau_{A}\right)$ is continuous. (This is a general property of inductive limits; cf. [12, p. 159].)

Let $\left\{f_{k}\right\}$ be a sequence convergent to a function $f$ in the space $\mathcal{D P}\left(\left\{E_{n}\right\}\right)$. This means that $p_{n}\left(F_{k}-F\right) \rightarrow 0$ for any fixed $n$ as $k \rightarrow \infty$. For some $n$ the set $E_{n}$ contains both $a$ and $b$. But then

$$
\omega_{F-F_{k}}([a, b]) \leq \operatorname{Var}\left(F_{k}-F, E_{n}\right)=p_{n}\left(F_{k}-F\right) \rightarrow 0
$$

so that $F_{k}$ converges uniformly to $F$. But that is convergence in the space $\left(\mathcal{D P}[a, b], \tau_{A}\right)$ and so $G\left(f_{k}\right) \rightarrow G(f)$ verifying that $G$ is continuous. Since $G$ is continuous the topology $\tau$ is finer than the topology $\tau_{A}$.

We now prove the following statement:

A linear functional $\Gamma$ on the space $(\mathcal{D P}[a, b], \tau)$ is continuous if and only if there is a function $g$ on $[a, b]$ that is equivalent to a function of bounded variation so that $\Gamma(f)=\int_{a}^{b} f(t) g(t) d t$ where the integral is in the Denjoy-Perron sense.

Suppose first that $\Gamma$ is a continuous linear functional on the space $\mathcal{D} \mathcal{P}[a, b]$ furnished with the topology $\tau$. Being continuous its restriction to the spaces $\mathcal{D P}\left(\left\{E_{n}\right\}\right)$ is also continuous. Thus we know from Theorem 3.2 that the equation

$$
\Gamma(f)=\int_{a}^{b} f(t) g(t) d t
$$

must hold for some $g$, but that $g$ may vary since that theorem applies to the separate subspaces. It is clear however that such a $g$ if it exists is unique up to a set of measure zero and so we know there is some bounded, measurable function $g$ for which the identity (20) holds for all $f \in \mathcal{D} \mathcal{P}[a, b]$. But the existence of the integral for all Denjoy-Perron integrable functions $f$ requires that $g$ be equivalent to a function of bounded variation.

Conversely suppose that $\Gamma(f)=\int_{a}^{b} f(t) g(t) d t$ does hold for all $f \in \mathcal{D P}[a, b]$ where $g$ is equivalent to a function of bounded variation. Then this is a continuous linear functional on the space $\mathcal{D P}[a, b]$ furnished with the Alexiewicz 
norm topology $\tau_{A}$. Since the topology here on $\mathcal{D P}[a, b]$ is finer than the topology generated by the Alexiewicz norm this function is also a continuous linear functional on this space.

Now we see that the space $\mathcal{D} \mathcal{P}[a, b]$ furnished with the Alexiewicz norm or furnished with the $\tau$ topology has the same family of continuous linear functionals. It is then a consequence of the Mackey-Arens theorem (see, for example, [21, pp. 131-132] or [12, p. 205]) that this requires that the Alexiewicz norm generates a finer topology than the $\tau$ topology (indeed $\tau_{A}$ must generate the finest topology having this class of continuous linear functionals). But we already noted that $\tau$ is finer than $\tau_{A}$. This proves the equivalence of the two topologies.

As a final remark we recall our worry that the Alexiewicz norm may not supply the most natural topology for this space. In light of this theorem it seems that it does.

\section{References}

[1] A. Alexiewicz, Linear functionals on Denjoy integrable functions, Colloq. Math., 1 (1948), 289-293.

[2] B. Bongiorno, W. F. Pfeffer, and B. S. Thomson, A full descriptive definition of the gage integral, Canadian Math. Bull., 39(4) (1996), 390-401.

[3] B. Bongiorno, L. Di Piazza and V. Skvortsov, A new full descriptive characterization of the Denjoy-Perron integral, Real Anal. Exch., Vol. 21, No. 2, (1995-1996) 656-663.

[4] B. Bongiorno, L. Di Piazza and D. Preiss, Infinite variation and derivatives in $\mathbb{R}^{n}$, Journal of Math. Anal. Appl., 224 (1998) 22-33.

[5] B. Bongiorno, T. V. Panchapagesan, On the Alexiewicz topology of the Denjoy space, Real Anal. Exch., Vol. 21, (1995-1996).

[6] A. M. Bruckner, Differentiation of Real Functions, Springer-Verlag (1978).

[7] A. M. Bruckner, J. B. Bruckner and B. S. Thomson, Real Analysis Prentice-Hall (1996).

[8] Z. Buczolich and W. F. Pfeffer, Variations of additive functions, Czech. Math. J., 47 (122) (1997) 525-555. 
[9] V. Ene, Real functions - current topics, Lect. Notes in Math., vol. 1603, Springer-Verlag, 1995.

[10] V. Ene, Characterizations of $V B^{*} G \cap(N)$, Real Analysis Exchange, 23 (1997-98) 571-600.

[11] V. Ene, Thomson's variational measure, Real Anal. Exch., 24 (1998-99) $523-565$.

[12] J. Horvath, Topological Vector Spaces and Distributions, Academic Press (1966).

[13] W. F. Pfeffer, The Riemann Approach to Integration: Local Geometric Theory. Cambridge University Press (1993).

[14] W. F. Pfeffer, The generalized Riemann-Stieltjes integral, Real Anal. Exch., Vol. 21, No. 2, 521-547.

[15] W. F. Pfeffer, On variations of functions of one real variable. Math. Univ. Carolin., 38 (1997) 61-71.

[16] W. F. Pfeffer and B.S. Thomson, Measures defined by gages, Canad. Journal of Math. 44 (6) 1992, 1303-1316.

[17] S. Saks, Theory of the Integral, Dover, (1937).

[18] W. L. C. Sargent, On the integrability of a product, J. London Math. Soc., 23 (1948), 28-34

[19] W. L. C. Sargent, On the integrability of a product. II, J. London Math. Soc., 26 (1951), 278-285.

[20] W. L. C. Sargent, On some theorems of Hahn, Banach and Steinhaus, J. London Math. Soc., 28 (1953), 438-451.

[21] H. H. Schaeffer, Topological Vector Spaces, MacMillan (1966).

[22] B. S. Thomson, Spaces of conditionally integrable functions, J. London Math. Soc., 2 (1970), 358-360.

[23] B. S. Thomson, Derivates of Interval Functions, Memoir Amer. Math. Soc., 452, Providence, 1991.

[24] B. S. Thomson, $\sigma$-finite Borel measures on the real line. Real Anal. Exch., Vol. 23, No. 1, (1997-98) 185-192. 